\title{
Search for tau neutrinos at PeV energies and beyond with the MAGIC telescopes
}

\author{
D. Góra ${ }^{1 *}$, M. Manganaro' ${ }^{2,3}$, E. Bernardini ${ }^{4,5}$, M. Doro ${ }^{6}$, M. Will ${ }^{2,3}$, S. Lombardi ${ }^{7}$, J. \\ Rico $^{8}$, D. Sobczynska ${ }^{9}$, for the MAGIC Collaboration ${ }^{\dagger}$ \\ E-mail: Dariusz.Gora@ifj.edu.pl
}

${ }^{1}$ Institute of Nuclear Physics Polish Academy of Sciences, PL-31342 Krakow, Poland

${ }^{2}$ Inst. de Astrofisica de Canarias, E-38200 La Laguna, Tenerife, Spain

${ }^{3}$ Universidad de La Laguna, Dpto. Astrof'isica, E-38206 La Laguna, Tenerife, Spain

${ }^{4}$ Deutsches Elektronen-Synchrotron (DESY), D-15738 Zeuthen, Germany

${ }^{5}$ Humboldt University of Berlin, Institut f'ur Physik Newtonstr. 15, 12489 Berlin Germany

${ }^{6}$ Universita di Padova and INFN, I-35131 Padova, Italy

${ }^{7}$ INAF National Institute for Astrophysics, I-00136 Rome,Italy

${ }^{8}$ Institut de Fisica d'Altes Energies (IFAE), The Barcelona Institute of Science and Technology,

Campus UAB, 08193 Bellaterra (Barcelona), Spain

${ }^{9}$ University of Lódz, PL-90236 Lodz, Poland

The MAGIC telescopes, located at the Roque de los Muchachos Observatory (2200 a.s.1.) in the Canary Island of La Palma, are placed on the top of a mountain, from where a window of visibility of about $5 \mathrm{deg}$ in zenith and $80 \mathrm{deg}$ in azimuth is open in the direction of the surrounding ocean. This permits to search for a signature of particle showers induced by earth-skimming cosmic tau neutrinos in the PeV to EeV energy range arising from the ocean. We have studied the response of MAGIC to such events, employing Monte Carlo simulations of upward-going tau neutrino showers. The analysis of the shower images shows that air showers induced by tau neutrinos can be discriminated from the hadronic background coming from a similar direction. We have calculated the point source acceptance and the expected event rates, for a sample of generic neutrino fluxes from photo-hadronic interactions in AGNs. The analysis of about 30 hours of data taken toward the sea leads to a point source sensitivity for tau neutrinos at the level of the down-going point source analysis of the Pierre Auger Observatory, if the AUGER observation time is dedicated to a similar amount by MAGIC.

EPS-HEP 2017, European Physical Society conference on High Energy Physics

5-12 July 2017

Venice, Italy

\footnotetext{
* Speaker.

${ }^{\dagger}$ https://magic.mpp.mpg.de/acknowledgements_19_05_2017.html
} 


\section{Introduction}

The discovery of an astrophysical flux of high-energy neutrinos by IceCube [1] was a major step forward in the ongoing search for the origin of cosmic rays, since neutrino emission by usually follows from hadronic interaction in astrophysical accelerators. The composition of neutrino flux at Earth is consistent with equal fractions of all neutrino flavors, though with large uncertainty $[2,3]$. Of particular interest is the identification of $v_{\tau}$, which is only expected to be produced in negligible amounts in astrophysical accelerators, but should appear in the flux detected by IceCube due to neutrino flavor oscillation. Up to now, there has been no clear identification of $v_{\tau}$ at high energies, due to their resemblance with signals induced by $v_{e}$ in ice/water detectors. However, the detection of $v_{\tau}$ is very important from both the astrophysical and particle physics point of view.

A conventional approach for the detection of neutrinos with energies in the $\mathrm{PeV}$ range is based on detectors which use large volumes of ice (IceCube) or water (ANTARES). They sample Cherenkov light from muons produced by muon neutrinos, or from electron and tau lepton induced showers initiated by the charged current interactions of electron and tau neutrinos. An alternative technique is based on the proposed observation of upward going extensive air showers produced by the leptons originating from neutrino interactions below the surface of the Earth, the so-called earth-skimming method $[4,5]$.

In this paper we study the possibility to use the MAGIC (Major Atmospheric Gamma Imaging Cherenkov) telescopes to search for air showers induced by tau neutrinos ( $\tau$-induced showers) in the PeV-EeV energy range. MAGIC is a system of two IACTs located at the Roque de los Muchachos Observatory (28.8 $\left.\mathrm{N}, 17.9^{\circ} \mathrm{W}\right)$, in the Canary Island of La Palma (Spain). They are placed $85 \mathrm{~m}$ apart, each with a primary mirror of $17 \mathrm{~m}$ diameter. The MAGIC telescopes, with a field of view (FOV) of $3.5^{\circ}$, have been built to detect cosmic $\gamma$-rays in the energy range $50 \mathrm{GeV}$ $50 \mathrm{TeV}[6]$.

In order to use MAGIC for tau neutrino searches, the telescopes need to be pointed in the direction of the tau neutrinos escaping first from the Earth crust and then from the ocean, i.e. at the horizon or a few degrees below. In such cases the telescopes can monitor a large volume in their FOV resulting in a space angle area (defined as the intersection of the telescopes FOV and the sea surface) of about a few $\mathrm{km}^{2}$. In [7], the effective area for up-going tau neutrino observations with the MAGIC telescopes was calculated analytically and found to reach $\sim 10^{3} \mathrm{~m}^{2}$ (at $100 \mathrm{TeV}$ ) and $10^{5} \mathrm{~m}^{2}$ (at $1 \mathrm{EeV}$ ) for an observation angle of about $1.5^{\circ}$ below the horizon, rapidly diminishing with higher inclination.

From an observational point of view, it is worth noting that the time that can be dedicated to this kind of observations almost does not interfere with regular MAGIC gamma-ray observations of the sky, because the sea can be pointed even in the presence of optically thick clouds above the MAGIC site (high clouds). In fact, high-altitude clouds prevent the observation of gamma-ray sources but still allow pointing the telescopes to the horizon. For the MAGIC site there are up to about 100 hours per year available, when high clouds are present.

\section{MAGIC observations and Monte Carlo simulations}

The MAGIC telescopes have taken data at very large zenith angles $\left(85^{\circ}<\theta<95^{\circ}\right)$ in the direction of the sea (seaON), slightly above the sea (seaOFF) and towards the Roque de los Mucha- 

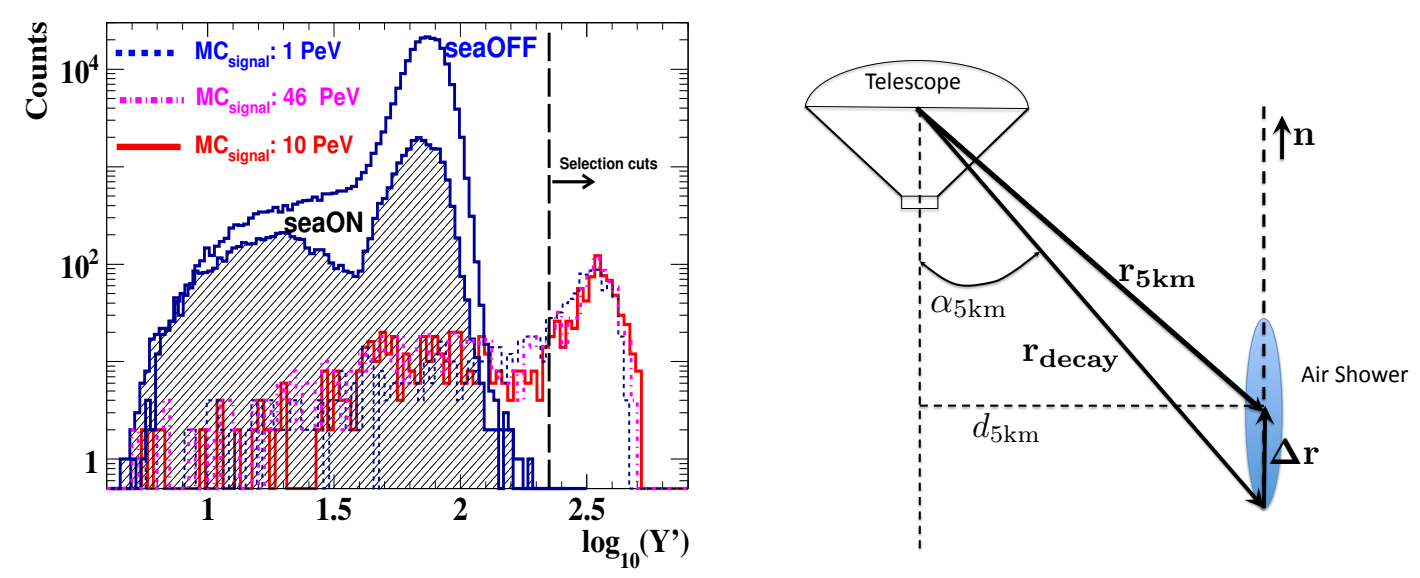

Figure 1: Left panel: The one-dimensional distribution of seaON, seaOFF and signal MC. The coordinate of events were obtained from the following formula: $\log _{10}\left(Y^{\prime}\right)=\log _{10}($ Size $[$ p.e. $]) * \cos (\alpha)-$ $\log _{10}($ Length $[\mathrm{deg}]) * \sin (\alpha)$, where $\alpha=63.435^{\circ}$. Note that above our selection cut $\left(\log _{10}\left(Y^{\prime}\right)>2.35\right)$ zero neutrino candidates are found. For showers with the larger impact distances $(0.3-1.3 \mathrm{~km})$ a slightly relaxed cut was used: $\log _{10}\left(Y^{\prime}\right)>2.10$; Right panel: Sketch illustrates the FOV cut used in our analysis, see text for more details.

chos mountain. The seaON data were taken at a zenith angle of $\theta=92.5^{\circ}$ while seaOFF was taken at $\theta=87.5^{\circ}$. The rate of stereo seaOFF events is about 27 times larger $(\sim 4.6 \mathrm{~Hz})$ than for seaON $(\sim 0.17 \mathrm{~Hz})$ observations. Thus 9.2 hours of seaOFF data provide high-statistics background estimates for about 30 hours of seaON data. It is worth to mention, that the $91 \%$ of the data were taken during nights characterized by optically thick high cumulus clouds, when normal $\gamma$-ray observations are usually worthless.

In order to study the signatures expected from neutrino-induced showers by MAGIC, a full Monte Carlo (MC) simulation chain was set up, which consists of three steps. First, the interaction of a given neutrino flux with the Earth and propagation of the resulting charged lepton through the Earth and the atmosphere is simulated using an extended version [8] of the ANIS code [9]. Second, the shower development of $\tau$-induced showers and their Cherenkov light production is simulated with CORSIKA [10]. The results of the CORSIKA simulation are used as inputs for the last step, i.e. the simulation of atmospheric extinction and the MAGIC detector response [11], see [12] for more details.

Each simulated event recorded and calibrated consists of a number of photoelectrons per camera pixel, which has been extracted using a sliding window algorithm [6]. In order to get rid of pixels whose contents are likely due only to night sky background (NSB) an image cleaning is performed. The resulting cleaned shower image contains only the pixels considered to obtain physical information about the shower. The cleaned camera image is characterized by a set of image parameters introduced by M. Hillas in [13]. These parameters provide a geometrical description of the images of showers and are used to infer the energy of the primary particle, its arrival direction, and to distinguish between $\gamma$-ray and hadron induced showers. In [12] we study these parameters for the case of deep $\tau$-induced simulated showers and compare the corresponding distributions with data. It is interesting that only with two Hillas parameters i.e. like Size and Length, we can easily 
identify a region with no background events, and construct a selection cut to identify tau-induced showers, see again [12] for more details. Here, we only show the one-dimensional distribution of seaOFF, seaON and MC signal simulation projected onto the line perpendicular to our selection cut, see Figure 1 (left panel). As can be seen we did not find any neutrino candidate, if the selection cut is applied to all seaON data. This plots shows that MAGIC can discriminate deep $\tau$-induced showers from the background of hadronic showers at high zenith angles.

\section{Monte Carlo estimate of MAGIC acceptance}

As previously mentioned the propagation of a given neutrino flux through the Earth and the atmosphere is simulated using an extended version of the ANIS code. Based on these simulations, the detector aperture/acceptance for an initial neutrino energy $E_{v_{\tau}}$ can be calculated from: $A^{\mathrm{PS}}\left(E_{V_{\tau}}, \theta, \phi\right)=N_{\text {gen }}^{-1} \times \sum_{i=1}^{N_{\text {Fovcut }}} P_{i}\left(E_{V_{\tau}}, E_{\tau}, \theta\right) \times A_{i}(\theta) \times T_{\text {eff }, i}\left(E_{\tau}, r_{5 \mathrm{~km}}, d, \theta\right)$, where $\theta, \phi$ are the simulated zenith and azimuth pointing angles of the MAGIC telescope, $N_{\text {gen }}$ is number of neutrino events from the direction $\theta$ and $\phi . N_{\mathrm{FOV} \text { cut }}$ is the number of $\tau$ leptons with energies $E_{\tau}$ larger than the threshold energy $E_{\mathrm{th}}=1 \mathrm{PeV}$ and with an estimated position of the shower maximum in the FOV of the MAGIC telescope, see Figure 1 (right panel) for an illustration of the geometry.

In addition the impact distance $d$ of the $\tau$-lepton induced showers is required to be smaller than $1.3 \mathrm{~km} . P\left(E_{v_{\tau}}, E_{\tau}, \theta\right)$ is the probability that a neutrino with energy $E_{v_{\tau}}$ and zenith angle $\theta$ produces a lepton with energy $E_{\tau} . A_{i}(\theta)$ is the physical cross-section area of the interaction volume seen by the neutrino, simulated by a cylinder with radius of $50 \mathrm{~km}$ and height $10 \mathrm{~km}$. $T_{\text {eff }, i}\left(E_{\tau}, r_{5 \mathrm{~km}}, d, \theta\right)$ is the trigger and reconstruction/cut efficiency for $\tau$-lepton induced showers with its estimated position of the shower maximum at distance $r_{5 \mathrm{~km}}$ from the telescope and the shower impact distance $d$. The trigger efficiency depends on the response of a given detector and is usually estimated based on MC simulations, see [12] for more details.

For shower energies relevant in this analysis i.e. 1-1000 PeV, the largest longitudinal extension of the shower is reached approximately after $600 \mathrm{~g} / \mathrm{cm}^{2}[14]^{1}$. Assuming that tau-induced showers develop in the densest part of the atmosphere with the near ground density of air of about $\rho_{\text {air }}=$ $0.0012 \mathrm{~g} / \mathrm{cm}^{3}$, this depth interval corresponds to about $\Delta r=5 \mathrm{~km}$. Thus, a position of the shower maximum can be estimated from the simple formula: $\vec{r}_{5 \mathrm{~km}}=\vec{r}_{\text {decay }}+\Delta r \cdot \vec{n}$, where $\vec{r}_{\text {decay }}$ is the vector pointing from the telescope to the decay vertex of the tau lepton and $\vec{n}$ is the unit vector describing the direction of the shower. In our FOV cut, we also included the fact that Cherenkov light has an opening angle of about $\alpha_{\text {Cher. }}=1.35^{\circ}$ for inclined directions, thus the Cherenkov light still can hit both mirror and camera even if its direction is outside the $1.75^{\circ}$ cone opened by the FOV of the MAGIC telescope. The following FOV condition was used: $\alpha_{5 \mathrm{~km}}=\arcsin \left(d_{5 \mathrm{~km}} / r_{5 \mathrm{~km}}\right)<$ $\left(\vartheta_{F O V} / 2+\alpha_{\text {Cher. }}\right) \approx 3.10^{\circ}$, where $d_{5 \mathrm{~km}}$ is the distance of the estimated shower maximum to the shower axis and $\vartheta_{F O V}=3.5^{\circ}$ is the FOV of MAGIC camera.

In Figure 2 (left panel) we show an estimate of the MAGIC point-source aperture (for $T_{\mathrm{eff}, i}=1$ ) to tau neutrinos. The aperture is shown for two cases: (1) for simulations including the orography of the La Palma island, but with the spherical model of Earth, with the rock density of about 2.65 $\mathrm{g} / \mathrm{cm}^{2}$, outside the island, and with the $\alpha_{r_{5 \mathrm{~km}}}<3.1^{\circ}$ cut; (2) for simulations including the orography

\footnotetext{
${ }^{1}$ In this approach shower-to-shower fluctuations are not included, which for a single shower can lead to the slightly different $\Delta r$.
} 

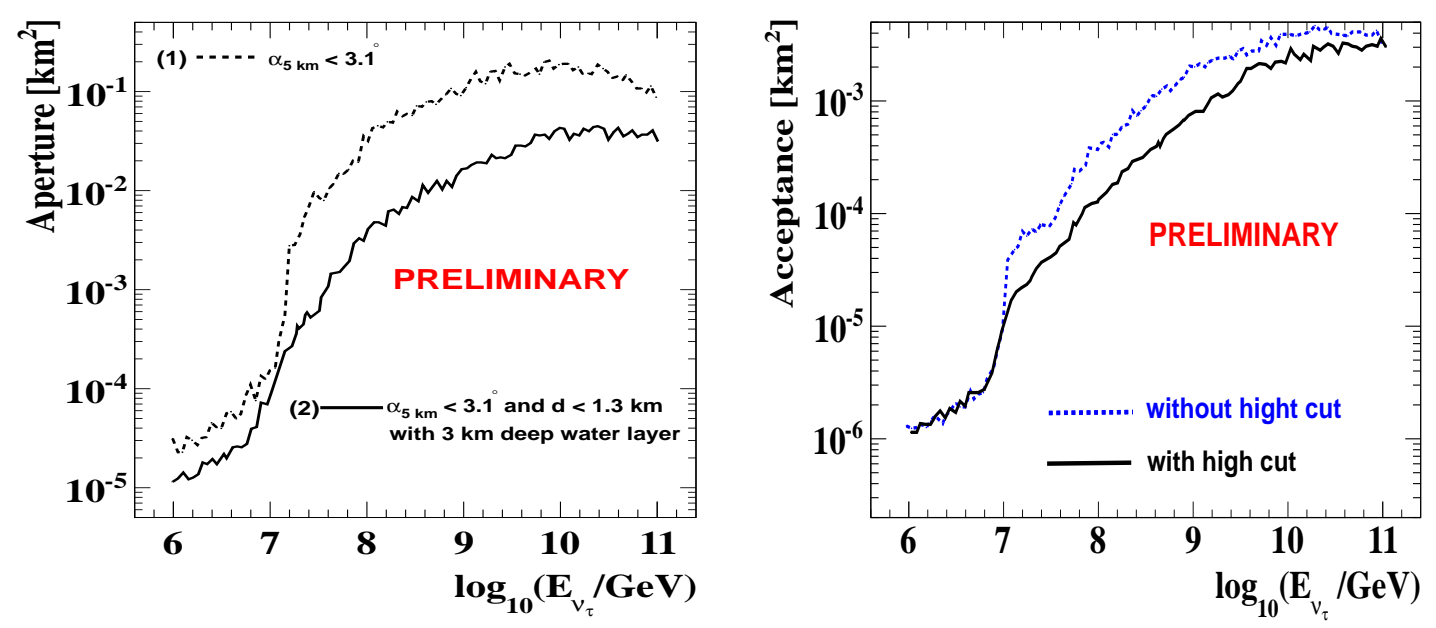

Figure 2: Left panel: The point-source aperture to earth-skimming tau neutrinos for the MAGIC telescopes pointing at $\theta_{\text {MAGIC }}=92.5^{\circ}$ and $\phi_{\text {MAGIC }}=-30^{\circ}$. Computations including the orographic conditions of the MAGIC site and for different densities of the interaction medium are shown (see text for more details); Right panel: $\mathrm{MC}$ acceptance for point sources, $A^{\mathrm{PS}}\left(E_{v_{\tau}}\right)$, for earth-skimming tau neutrinos, as estimated for the MAGIC site, with a FOV cut of: $\alpha_{5 \mathrm{~km}}<3.1^{\circ}$ and $d<1.3 \mathrm{~km}$ and within identification efficiencies shown in [12]. Note the significant influence of the heigh cut on the calculated acceptance.

of the La Palma island [15] and the $3 \mathrm{~km}$ deep water layer around the La Palma island. As we can see in the plot, the water layer is important because it leads to about a factor two (in the energy range 10-100 PeV) smaller aperture than for the spherical Earth calculations with a rock density of about $2.65 \mathrm{~g} / \mathrm{cm}^{2}$ outside the La Palma island. This is because the mixture of a first dense material (rock) and then much less dense one produces many tau leptons which then decay inside the water and are lost for observations. Another important effect, which was also included in our calculations are possible clouds during observations. We can have information from the MAGIC lidar system [16], whenever high clouds in the vertical direction are present at the MAGIC site. However, we did not have any information about clouds present in the direction of the seaON and seaOFF observations, due to the lack of possible data measurement in these directions. Thus, in our acceptance calculations we include the quasi-stable sea of cumulus between 1500 and $1900 \mathrm{~m}$ a.s.l., usually present at the MAGIC site due to the temperature inversion layer at these altitudes. To estimate the effect of this we assumed that all decaying tau leptons below 1500 a.s.l. are discarded in the acceptance calculations i.e. in $N_{\text {FOV cut }}$. We assumed that for such a case the Cherenkov light is absorbed when it passes the layer from 1.5-1.9 km a.s.l. Such a cut provides a conservative upper limit on this effect. In Figure 2 (right panel) we show the acceptance, when our selection cut, identification efficiency and the height cut is included in our simulations. As we can see in the plot the height cut leads also to a smaller (about factor two) acceptance.

\section{Event rate and tau neutrino sensitivity}

Object like flaring sources, including GRBs, AGNs, Tidal disruption or the Low Luminosity GRB (LLGRBS), can provide a boosted flux of neutrinos. Thus, we provide an estimate of the 

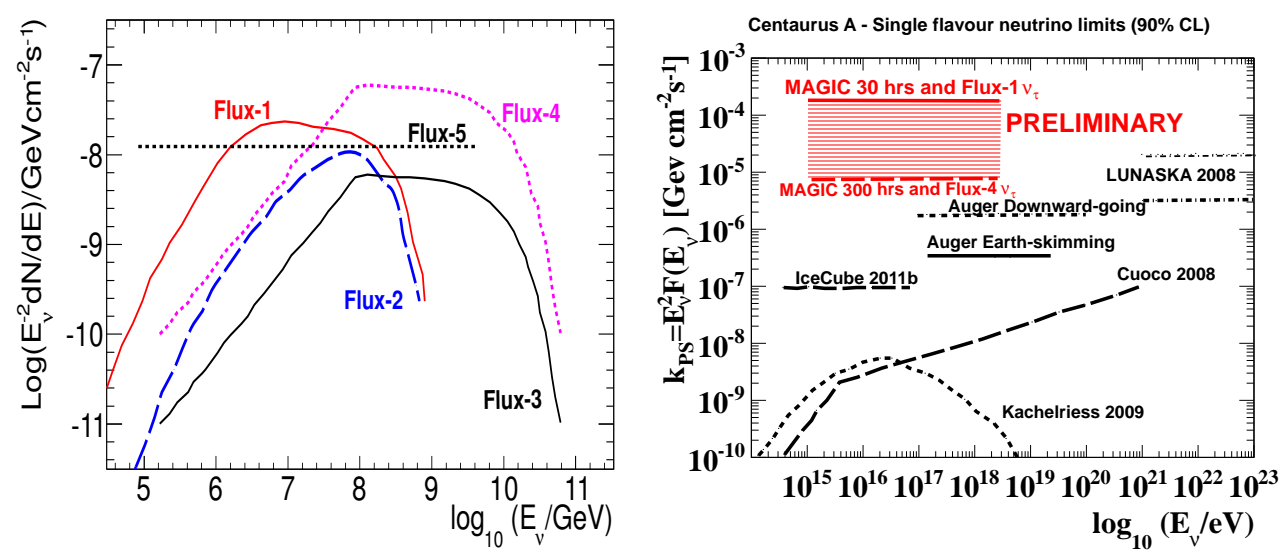

Figure 3: Left panel: A sample of representative neutrino flux predictions from photo-hadronic interactions in AGNs. Flux-1 and Flux-2 are calculations for the $\gamma$-ray flare of 3C 279 [17]. Flux-3 and Flux-4 represent predictions for PKS 2155-304 [18]. Flux-5 corresponds to a prediction for 3C 279 [19]. Right panel: The Pierre Auger upper limits at $90 \%$ C.L. on a single flavor neutrino flux from the active galaxy Centaurus A from the Earth-skimming and downward-going neutrino analysis [21]. The MAGIC point-source sensitivity for 30/300 hours is marked by red solid lines and the hatched area. Note the different energy range for MAGIC and the Pierre Auger Observatory. Plot adopted from [21].

event rate for a sample of generic neutrino fluxes, from photo-hadronic interactions in case of flaring AGNs, if observed within the MAGIC observation window. In Table 1 the expected event rates for MAGIC are shown for fluxes from the AGN benchmark models shown in Figure 3 (left panel). The rate is calculated for tau neutrinos assuming that the source is in the MAGIC telescope FOV for a period of 3 hours and with the acceptance calculated using the height cut/without height cut. For Flux-3 and Flux-4 the event rate is at the level of $3 \times 10^{-5}$ (i.e. those models covering the energy range beyond $\sim 1 \times 10^{8} \mathrm{GeV}$ and after application of the height cut). For neutrino fluxes covering the energy range below $\sim 5 \times 10^{7} \mathrm{GeV}$ (Flux-1, Flux-2, Flux-5), the number of expected events is not larger than $1.1 \times 10^{-5}$. In case of no height cut applied, the number of expected events is about a factor of two larger.

From the estimated acceptance with the height cut, the sensitivity for an injected spectrum $K \times \Phi\left(E_{v}\right)$ with a known shape $\Phi\left(E_{v}\right)$ was calculated. The $90 \%$ C.L. on the value of $K$, according to [20] is $K_{90 \%}=2.44 / N_{\text {Events, }}$, with the assumption of negligible background, zero neutrino events being observed by the MAGIC during sea observations, and in case of an assumed flux of $\Phi\left(E_{v}\right)=$

Table 1: Expected event rates for the MAGIC detector in case of AGN flares. Case A: simulations with the height cut included, case B without the height cut.

\begin{tabular}{ccccccc}
\hline \hline & & $\begin{array}{c}\text { Flux-1 } \\
\left(\times 10^{-5} / 3 \text { hrs }\right)\end{array}$ & $\begin{array}{c}\text { Flux-2 } \\
\left(\times 10^{-5} / 3 \text { hrs }\right)\end{array}$ & $\begin{array}{c}\text { Flux-3 } \\
\left(\times 10^{-5} / 3 \text { hrs }\right)\end{array}$ & $\begin{array}{c}\text { Flux-4 } \\
\left(\times 10^{-5} / 3 \text { hrs }\right)\end{array}$ & $\begin{array}{c}\text { Flux-5 } \\
\left(\times 10^{-5} / 3 \text { hrs }\right)\end{array}$ \\
\hline \hline$N_{\text {Events }}$ & case A & 2.4 & 1.4 & 0.74 & 7.4 & 2.4 \\
$N_{\text {Events }}$ & case B & 1.1 & 0.6 & 0.30 & 2.9 & 1.2 \\
\hline \hline
\end{tabular}


$1 \times 10^{-8} E^{-2}\left[\mathrm{GeV} \mathrm{cm}^{-2} \mathrm{~s}^{-1}\right]$, the $90 \%$ C.L. limit for a point source search is then:

$$
E_{V_{\tau}}^{2} \Phi^{P S}\left(E_{V_{\tau}}\right)<2.0 \times 10^{-4}\left[\mathrm{GeV} \mathrm{cm}^{-2} \mathrm{~s}^{-1}\right]
$$

in the range from 2 to $1000 \mathrm{PeV}$. The sensitivity is calculated for the expected number of tau neutrino events equal to $N_{\text {Events }}=1.2 \times 10^{-4}$, based on the result listed in Table 1 for Flux-5, and for 30 hours of observation time. The limit can be improved about one order of magnitude for larger observation times, such as the expected event rate in case of a strong flare. As it is seen from Table 1 for Flux-4, and the observation time of $\sim 300 \mathrm{hrs}$ i.e. the expected number of tau neutrino events is $N_{\text {Events }}=2.9 \times 10^{-3}$, thus the $90 \%$ C.L. limit could reach the value of $E_{v_{\tau}}^{2} \Phi^{P S}\left(E_{v_{\tau}}\right)<8.4 \times 10^{-6}\left[\mathrm{GeV} \mathrm{cm}^{-2} \mathrm{~s}^{-1}\right]$ i.e. about factor two lower than the limit of the downgoing analysis of the Pierre Auger Observatory [21], see Figure 3 (right panel). Note that the Auger downward limit corresponds to an equivalent exposure of about $\sim 17520 \mathrm{hrs}$, while MAGIC one (from Eq. 4.1) to only $30 \mathrm{hrs}$. If we take into account this difference then the MAGIC sensitivity for tau neutrinos is at the similar level like for the the down-going point source analysis of the Pierre Auger Observatory i.e. if observations will be made for the same number of hours.

\section{Summary}

We have presented results from MC simulations of $\tau$-induced air showers and MAGIC observations at very high zenith angles. In particular, we have calculated the point source acceptance and the expected event rates, for a sample of generic neutrino fluxes from photo-hadronic interactions in AGNs. Taking into account that for this purpose MAGIC has to be pointed below the horizon during moonless nights, the observational program for tau neutrino searches seems to be challenging, but in principle not impossible to pursue. In fact, a significant amount of observation time can be accumulated during periods with high clouds, when those instruments are usually not used for gamma-ray observations. This means that during a few IACTs observational seasons a significant amount of time can be accumulated. This makes the perspective for detection of tau neutrino induced showers by IACTs more attractive. With more than 1200 hours of data the achievable limit can reach the level of the results for down-going neutrinos of the Pierre Auger Observatory. Finally, the next-generation Cherenkov telescopes, i.e. the Cherenkov Telescope Array, will exploit their a larger FOV (in extended observation mode), and much larger effective areas.

\section{References}

[1] M.G. Aartsen, et al., Phys. Rev. Lett. 113101101 (2014) [arxiv1405.5303]

[2] M.G. Aartsen, et al., Phys. Rev. Lett. 114, 171102 (2015) [arXiv:1502 .03376]

[3] M.G. Aartsen, et al., Astrophysical Journal 809, 98 (2015) [arXiv:1507. 03991]

[4] D. Fargion, Astrophysical Journal 570, 909 (2002) [astro-ph 0002453 ]; D. Fargion, Prog. Part. Nucl. Phys. 57, 384 (2006)

[5] X. Bertou, et al., Astroparticle Physics 17, 183 (2002) [astro-ph/ 0104452 ]

[6] J. Aleksic, et al., Astroparticle Physics 72, 76 (2016) [arXiv: 1409 . 5594] 
[7] M. Gaug, et al., Proc of 30th I.C.R.C. (Merida) 1273 (2007) [arXiv: 0709.1462 ]

[8] D. Góra, M. Roth, A. Tamburro, Astroparticle Physics 26, 402 (2007)

[9] A. Gazizow and M. Kowalski, Comput. Phys. Commun. 172, 2013 (2005)

[10] D. Heck, J. Knapp, J.N. Capdevielle, G. Schatz, T. Thouw, Report FZKA 6019 (1998)

[11] R. Zanin, et al. 2013 Proc of 33rd I.C.R.C. (Rio de Janeiro) (2013)

[12] D. Góra, et al. for the MAGIC Collaboration, PoS(ICRC2017), 992 (2017) [ arXiv: 1708 . 06147 ]

[13] M.A. Hillas, Nucl. Phys. Proc. Suppl. 52B, 29, (1997)

[14] K.H. Kampert and M. Unger, Astroparticle Physics 35, 106602012 [ arXiv: 1201.0018 ]

[15] Consortium for Spatial Information (CGIAR-CSI) http://srtm.csi.cgiar.org/

[16] Ch. Fruck, et al. Proc of 33rd I.C.R.C. (Rio de Janeiro) (2013) [ arXiv: 1403.3591 ]

[17] A. Reimer, Int. Journ. of Mod. Phys. D 181511 (2009)

[18] J.K. Becker, et al., Nucl. Instr. and Meth. in Phys. Res. Sect. A 630, 1, 269 (2011)

[19] A. Atoyan, C.D. Dermer, Phys. Rev. Lett. 87, 221102 (2001)

[20] G.J. Feldman, R.D. Cousins, Phys. Rev. $D 573889$ (1998)

[21] P. Abreu, et al., The Astrophysical Journal Letters 755, L4 [ arXiv: 1210 . 3143] 